\title{
CCR-5 D32 and SDF-I 3' A Polymorphism In Exposed But Uninfected Partners of HIV Infected Individuals in North India P Suresh*‡ and A Wanchu*
}

\author{
Address: Department of Internal Medicine, PGIMER, Chandigarh, India \\ Email: P Suresh* - suresh_pallikkuth@yahoo.com \\ * Corresponding authors $\ddagger$ Presenting author
}

from 2005 International Meeting of The Institute of Human Virology

Baltimore, USA, 29 August - 2 September 2005

Published: 8 December 2005

Retrovirology 2005, 2(SuppI I):P77 doi: 10.I I86/1742-4690-2-SI-P77

\section{Background}

Genetic variations in chemokine genes have been associated with protection against infection by HIV-1 or slow progression to AIDS in infected individuals. We carried out this study to determine the prevalence of polymorphisms in CCR-5 D32 and SDF-1 3' genes an in exposed but uninfected partners of HIV infected individuals in North India.

\section{Materials and methods}

25 exposed but uninfected (EU) individuals and 25 normal healthy controls (HC) with low risk of infection were studied. Genomic DNA was extracted from whole blood and PCR was performed for genotyping. CCR-5 D32 and SDF-1 3'A were studied by PCR-RFLP using EcoR I and Msp I enzyme, respectively.

\section{Results}

No CCR-5 D32 mutations were detected in any individual. Both EU and HC showed a wild type CCR-5 gene. SDF-1 3'A gene polymorphism was frequently detected. Variant allele frequencies were $36 \%$ in EU and 20\% in HC (P > 0.5). Out of 25 EU's, 16 (64\%) wild type, 8 (32\%) heterozygous and $1(4 \%)$ were homozygous were detected while in HC, 20 (80\%) wild type and 5 (20\%) heterozygous were detected. No homozygous mutation was detected in the control group.

\section{Conclusion}

CCR-5 D32 polymorphism, which was common among Caucasians, was not present in our population. Increased frequency of SDF-1 3'A polymorphism was detected in both EU's and controls. Additional factors must be oper-

ating in protecting exposed uninfected individuals in our population. 